\title{
Tongue: Anatomy, functions and orthodontic implications
}

\author{
Parijat Chakraborty $^{*}$, Richa Dhingra ${ }^{2}$, Pratik Chandra ${ }^{3}$, Ragni Tandon $^{4}$, Aftab Azam ${ }^{5}$, Ashish Chauhan ${ }^{6}$ \\ ${ }^{1,2}$ Post Graduate, ${ }^{3}$ Reader, ${ }^{4}$ Professor and HOD, ${ }^{5}$ Professor, ${ }^{6}$ Senior Lecturer, Dept. of Orthodontics and Dentofacial Orthopaedics, \\ Saraswati Dental College, Lucknow, Uttar Pradesh, India
}

*Corresponding Author: Parijat Chakraborty

Email: parijatchak@gmail.com

\begin{abstract}
Malocclusion can also be caused by abnormal functions, for instance, mouth breathing, tongue thrust, swallowing, and unilateral chewing and also by abnormal postures of oral circumferential muscles like forward tongue thrust, tongue biting, and low tongue at rest. Orthodontists agree that the tongue's habitually abnormal movement can cause a variety of malocclusions. The aim of the present literature is to enlighten the anatomy \& development of tongue, along with its functions and orthodontic implications (role in malocclusion).
\end{abstract}

Keywords: Tongue, Swallowing, Mastication, Orthodontics.

\section{Introduction}

Orthodontists agree that the tongue's habitually abnormal movement can cause a variety of malocclusions. It is universally acknowledged that the power of the tongue misshapes the arches and many concerned study has been devoted to determine whether the tongue is an important factor in malocclusion.

Malocclusion could also be caused by abnormal functions, for instance, mouth breathing, tongue thrust, swallowing, and unilateral chewing and also by abnormal postures of oral circumferential muscles like forward tongue thrust, tongue biting, and low tongue at rest. Forces from unintentional and habitual behaviors constantly working on the maxillofacial and alveolar regions can cause the bony structures to generally deform, bringing about jaw deformity and malocclusion.

The aim of the present literature is to enlighten the anatomy \& development of tongue, along with its functions and orthodontic implications.

\section{Anatomy of Tongue \& Development of Dental Arches (Fig. 1)}

The normal growth, development and function of the tongue is well integrated with the growth, development and function of all other related oral and dental structures which inevitably leads to normal jaw relation and dental function. Three theories have been developed regarding factors which augment inherent growth tendencies. These are summarized as follows:

1. The development of the jaws is due principally to occlusal force during mastication.

2. The development of the jaws is governed principally by the development of the teeth.

3. The development of the jaws is fostered principally by muscle activity

\section{Functions of Tongue (Chart 1) Mastication}

The tongue assistance in mastication is also well recognized. Besides controlling, with the assistance of the buccinator the position of the food between the teeth, it also turns the food and mixes it with saliva. On the other hand, it has been evident that the tongue significantly influences masticatory efficiency. The tongue movement with the mouth closed appears to be closer to movement during mastication than movement with the mouth open. Tongue movement showed the greatest difference compared to action potential with the mouth closed in the resting position involved muscle activity during lateral movement of the tongue.

Swallowing is an essential act similar to breathing or blood circulation. It begins during fetal life and corresponds to all the muscle and joint movements that allow passing the "bolus" (including saliva) in the oral cavity to the digestive tract. (Fig. 2)

It consists of three successive phases: oral, pharyngeal, esophageal. Oral phase of swallowing implements not only the lingual and masticatory muscles as well as the facial muscles and the infra-hyoid muscles and sub-hyoid. In the infant, swallowing moved from birth through nutrition function. The tongue is then in a low position and intervened between the arcades during swallowing. Daily frequency (between 1000 and 3000 times per day) and constantly repeated functional stimulation it causes, among other at the TMJ level, allows the progressive installation during growth, of an instantaneous anatomical correlation, between maximum occlusion and articular swallowing position. The role and position of tongue are essential to a physiological swallowing.

In children, in deciduous and mixed dentition, atypical or inaccurate lingual postures are consistently associated with facial deformities (Deffez 1995). Disorders of the position and volume lingual, dyskinesia thus directly affect facial growth and positioning of the teeth in occlusion and along occlusal curves (Wilson and Spee). 
Chart 1: Functions of the tongue
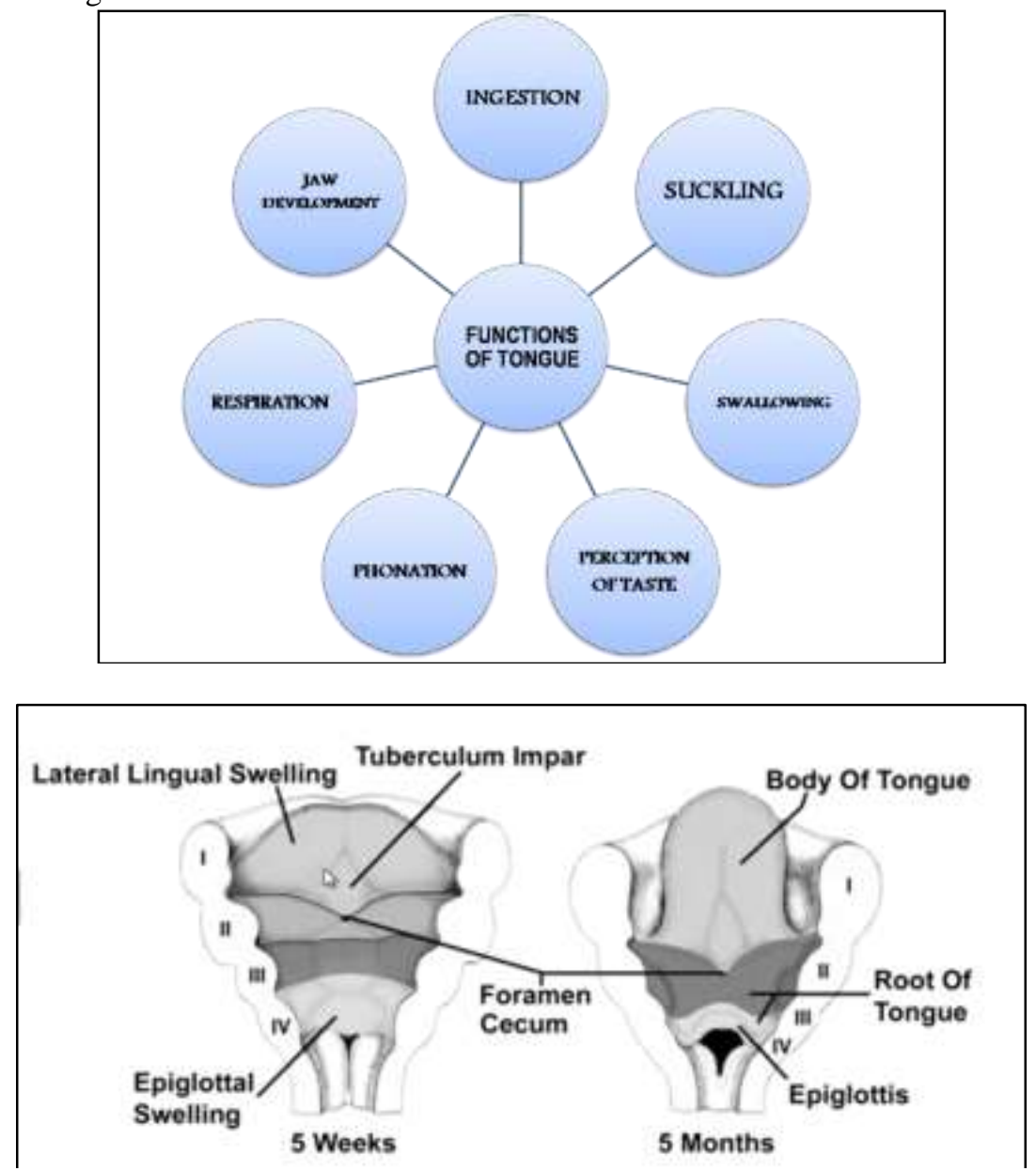

Fig. 1: Development of tongue

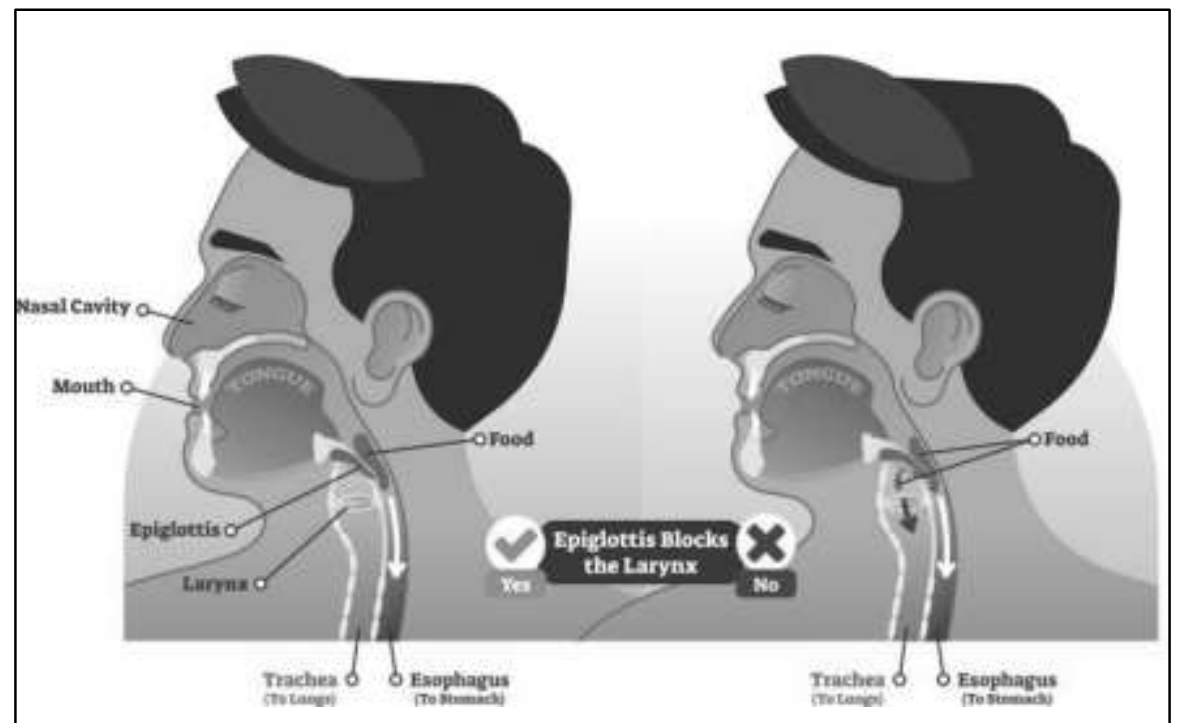

Fig. 2: Phases of swallowing

\section{Speech}

Tongue helps in the formation of sounds like $-\mathrm{s}, \mathrm{z}, \mathrm{t}, \mathrm{d}$, sh, $\mathrm{e}, \mathrm{g}$, is. Hence, it improves speech.

\section{Breathing}

While the tongue is at rest, nasal breathing occurs. But a habit or a force is always associated with forced mouth breathing. 
Equilibrium theory and development of dental occlusion Contributors to dental equilibrium-

1. Masticatory forces.

2. Soft tissue pressures from the lips, cheeks \& tongue.

3. External pressures- habits \& orthodontic forces.

4. Intrinsic pressures- gingival \& PDL fibres.

\section{Buccinator Mechanism (Fig. 3)}

Buccinator is a quadrilateral muscle between maxilla and mandible and it forms the mobile and adaptive substance of cheek.

1. Winders has shown that during mastication and deglutition, the tongue may exert 2 to 3 times as much force on the musculature as the lips and cheeks at any one time.

2. Role of buccinator mechanism is in maintaining arch form and teeth in position.

3. Starting with the decussating fibers of orbicularis oris muscle joining the right and left fibers within the lips, the Buccinator Mechanism runs laterally and posteriorly around the corners of mouth, joining other fibers of buccinator muscle which inserts into the pterygomandibular raphe just behind the dentition

4. Here, it intermingles with the fibers of superior constrictor muscle which continues medially and posteriorly to anchor at the pharyngeal tubercle of occipital bone.

5. The decussating fibers of orbicularis oris muscle, the anterior component of the buccinator mechanism.

6. Opposing the buccinator mechanism on the lingual aspect, is a very powerful muscle - the Tongue.

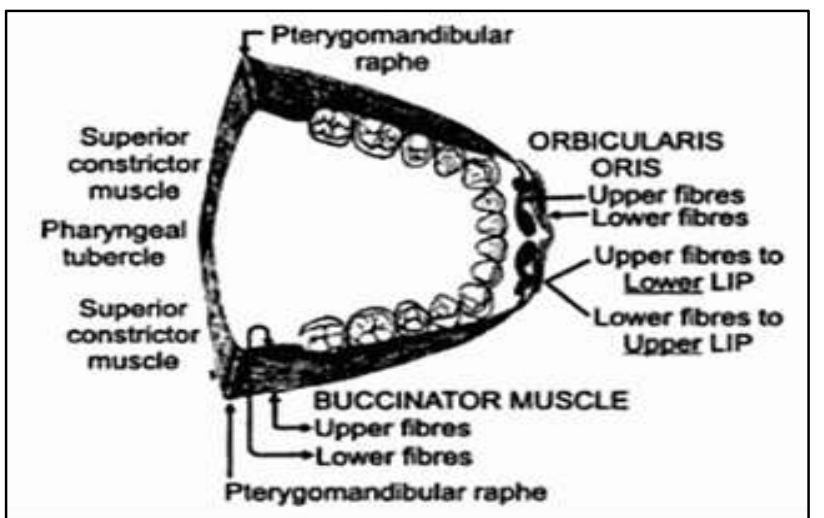

Fig. 3: Buccinator mechanism

\section{Orthodontic Implications \\ Examination of the tongue}

From orthodontic perspective, morphologic examination i.e. size and shape; functional examination (posture) are very important. Tongue asymmetry is important in determining dental arch symmetry, midlines, maintenance of treated incisal relations and even open bite. Functional examination is cephalometrically performed (metric evaluation),

\section{Role in malocclusion}

Genioglossus reflex (initiated by large tongue) causes sustained Jaw opening further resulting into sustained tongue posture, which leads to malocclusion like proclination, openbite, deepbite, etc.

Graber $(1975)^{6}$ in his book considered the influence of tongue position and tongue size along with the size of the mandible to be important factor in the dental arch and bone formation. In support to this literature, Naosuke Doto $(2015)^{7}$ hypothesized that the lip closing force and tongue pressure were very important for preventing malocclusion and achieving post orthodontic treatment stability. The abnormal morphology of the lower lip and tongue may be primary/ secondary to the malocclusion and may tend to produce deterioration of incisor relation after treatment. Lambrechts $(2010)^{8}$ put forward different roles of the internal and external muscular forces that were guiding eruption, influencing occlusal formation, and maintaining dental arch shape and stability.

According to the Theory of Tomes (1873), ${ }^{9}$ the perioral musculature and tongue principally determines the position of the teeth. Others authors acknowledged that muscle function, duration, speech and swallow can be a primary factor in causing and perpetuating a malocclusion.

The pressure from tongue on the dentition can be measured by various techniques like EMG, Cineradiography, Palatographic, Sensors, Balloon filled Pressure tips.

\section{Conclusion}

Hence, we can conclude that the position, size and function play a vital role in dental malocclusion. Also, habits like tongue thrusting trouble the orthodontists as relapse chances increases in such cases.

\section{Source of Funding}

None.

\section{Conflict of Interest}

None.

\section{References}

1. Swinehart DR. The importance of the tongue in the development of normal occlusion. Am J Orthod. 1950;36(11):813-30.

2. Yamaguchi H, Sueishi K. Malocclusion associated with abnormal posture. Bull Tokyo Dent Coll. 2003;44(2);43-54.

3. Cohen JT. Growth and Development of the Dental Arches in Children. J Am Dent Assoc. 1940;27(8):1250-60.

4. Abd-El-Malek S. The part played by the tongue in mastication and deglutition. J Anat. 1955;9(2):250-4.

5. Oguchi H, Watanabe T, Nakamura N, Watanabe S. Influence of tongue movements on masticatory efficiency. Dent Oral Craniofac Res. 2016;2(6):1-6.

6. Graber TM, Swain BF. Current orthodontic concepts and techniques. W.B. Saunders Company; 2nd edition (January 1, 1975). 
7. Doto N, Yamada K. The relationship between maximum lip closing force and tongue pressure according to lateral craniofacial morphology. Orthod Waves. 2015;74(3):69-75.

8. Helen Lambrechts, Evelyne De Baets, Steffen Fieuws, Guy Willems. Lip and tongue pressure in orthodontic patients. Eur J Orthod. 2010;32(4):466-71.
9. Tomes CS. The bearing of the development of the jaws on irregularities. Dent Cosmos. 1873;15:292-6.

How to cite: Chakraborty $\mathrm{P}$, Dhingra R, Chandra P, Tandon R, Azam A, Chauhan A. Tongue: Anatomy, functions and orthodontic implications. Indian J Orthod Dentofacial Res.2020;6(1):1-4. 